\title{
Effects of Prenatal Cocaine Exposure on Social Development in Mice
}

\author{
Zeeba D. Kabir ${ }^{a, b}$ Bruce Kennedy ${ }^{c}$ Aaron Katzman ${ }^{d}$ Garet P. Lahvis $^{e}$ \\ Barry E. Kosofsky ${ }^{a, b}$ \\ ${ }^{a}$ Department of Pediatrics, Division of Pediatric Neurology and ${ }^{b}$ Brain and Mind Institute, Weill Cornell Medical \\ College, New York, N.Y., ' Department of Neuroscience, University of Minnesota, Minneapolis, Minn., ${ }^{\mathrm{d}}$ Department

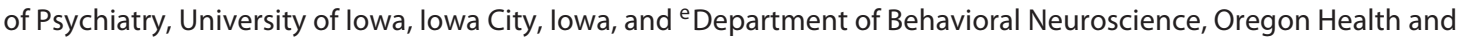 \\ Science University, Portland, Oreg., USA
}

\section{Key Words}

Medial prefrontal cortex - Prenatal cocaine exposure .

Social interaction · Ultrasonic vocalizations · Early growth response protein 1

\begin{abstract}
Prenatal cocaine exposure (PCE) in humans and animals has been shown to impair social development. Molecules that mediate synaptic plasticity and learning in the medial prefrontal cortex (mPFC), specifically brain-derived neurotrophic factor (BDNF) and its downstream signaling molecule, early growth response protein 1 (egr1), have been shown to affect the regulation of social interactions (SI). In this study we determined the effects of PCE on SI and the corresponding ultrasonic vocalizations (USVs) in developing mice. Furthermore, we studied the PCE-induced changes in the constitutive expression of BDNF, egr 1 and their transcriptional regulators in the MPFC as a possible molecular mechanism mediating the altered SI. In prenatal cocaine-exposed (PCOC) mice we identified increased SI and USV production at postnatal day (PD) 25, and increased SI but not USVs at PD35. By PD45 the expression of both social behaviors normalized in PCOC mice. At the molecular level, we found increased BDNF exon IV and egr 1 mRNA in the MPFC of PCOC mice at PD30 that normalized by PD45. This was concurrent with increased
\end{abstract}

EGR1 protein in the $\mathrm{MPFC}$ of $\mathrm{PCOC}$ mice at PD30, suggesting a role of egr 1 in the enhanced $\mathrm{SI}$ observed in juvenile PCOC mice. Additionally, by measuring the association of acetylation of histone 3 at lysine residues 9 and $14(\mathrm{acH} 3 \mathrm{~K} 9,14)$ and $\mathrm{MeCP} 2$ at the promoters of BDNF exons I and IV and egr1, our results provide evidence of promoter-specific alterations in the $\mathrm{MPFC}$ of PCOC juvenile mice, with increased association of acH3K9,14 only at the BDNF exon IV promoter. These results identify a potential PCE-induced molecular alteration as the underlying neurobiological mechanism mediating the altered social development in juvenile mice.

(c) 2014 S. Karger AG, Basel

\section{Introduction}

The development of limbic neurocircuits is sensitive to social environment and plays an important role in regulating social interactions (SI) $[1,2]$. Thus, drugs that interfere with the development of limbic circuits would be expected to influence the ability to regulate SI. Prenatal cocaine exposure (PCE) has been associated with deficits in the development of social behaviors in humans [3], with increased passive-withdrawn negative engagement in infants [4] and increased aggression among juveniles [5]. While rodent studies show that PCE can lead to defi-

\section{KARGER}

E-Mail karger@karger.com

www.karger.com/dne
(C) 2014 S. Karger AG, Basel

$0378-5866 / 14 / 0364-0338 \$ 39.50 / 0$
Barry E. Kosofsky, MD, PhD

Weill Cornell Medical College

525 East 68th Street, Box 91

New York, NY 10021 (USA)

E-Mail bar2009@med.cornell.edu 
cits in social behavior [6-9], the mechanisms that underlie the relationship between PCE and social regulation have not been clearly identified. In adult animals that were exposed to cocaine in utero several studies have reported depressed SI [6-8]. However, the effects of PCE on SI in juvenile animals show conflicting results. While Overstreet et al. [6] (2000) found that PCE depressed SI among juvenile rats tested at postnatal day (PD) 30, other similar studies of juveniles show no effect of treatment [9]. Furthermore, no study to date has evaluated how PCE contributes to molecular changes that might underlie SI dysregulation.

The medial prefrontal cortex (mPFC) plays an important role in SI regulation $[10,11]$. Molecules that mediate synaptic plasticity and learning in the mPFC, specifically the extracellular signal-regulated kinase 2 (ERK2) pathway and its downstream signaling molecule early growth response protein 1 (EGR1), have been shown to mediate SI $[12,13]$. ERK2 expression is regulated by brain-derived neurotrophic factor (BDNF) in the hippocampus [14]. Activation of BDNF in primary cortical cultures leads to the translocation of ERK2 into the nucleus where it activates the transcription of egr 1 [15]. These results suggest that BDNF signaling pathways within the $\mathrm{MPFC}$ may be impacting SI via regulation of ERK2 and egr1.

Of the nine unique transcripts comprising the BDNF gene, those containing exons I and IV are the most abundantly transcribed in the mPFC of mice [16]. Transcription of BDNF from exons I and IV, as well as egr 1, is dynamically regulated by changes in chromatin structure that is mediated in part by posttranslational modifications of histone proteins. Acetylation of histone 3 at lysine residues 9 and $14(\mathrm{acH} 3 \mathrm{~K} 9,14)$ acts as a marker of transcriptional activation as it results in an open chromatin configuration that increases accessibility of transcription factors to specific DNA promoter regions. Methyl cytosine-binding protein 2 (MeCP2) is one such transcription factor that regulates the transcription of BDNF exons I and IV, and egr 1, by altering its binding status at specific sites in their promoter regions [17-19]. In this study, we were interested in determining the effects of PCE on different aspects of the regulation of SI in juvenile (PD2535 ) and adolescent (PD45) mice and their production of ultrasonic vocalizations (USVs) during these interactions. Furthermore, we aimed to identify the effects of PCE on the constitutive expression of BDNF and EGR1 and their transcriptional regulators, specifically in the $\mathrm{mPFC}$, as a possible molecular mechanism mediating the altered SI.

Social Development in Prenatal

Cocaine-Exposed Mice

\section{Materials and Methods}

\section{Animals and Prenatal Treatments}

Wild-type male mice of Swiss Webster background were used for all experiments. A transplacental cocaine treatment regimen as previously described [20] was used to expose mouse embryos to cocaine. Adult timed-pregnant Swiss Webster dams were purchased from Taconic (Germantown, N.Y., USA) with each dam being assigned to one of two treatment groups and receiving twicedaily subcutaneous injections (at 7.00 a.m. and 7.00 p.m.) from E8 to E17, inclusive, of cocaine $\mathrm{HCl}$ (Sigma-Aldrich, St. Louis, Mo., USA; $20 \mathrm{mg} / \mathrm{kg} /$ injection, s.c., dissolved in saline) totaling $40 \mathrm{mg} /$ $\mathrm{kg}$ per day (prenatal cocaine-exposed offspring, PCOC) or $0.9 \%$ saline (prenatal saline-exposed offspring, PSAL). Though dams injected with cocaine gained less weight during gestation, there was no effect of PCE on the number of live born pups per litter (data not shown). Within $24 \mathrm{~h}$ of birth, all pups were surrogate fostered to control dams (Swiss Webster; Taconic Labs) that had delivered within the preceding 24-72 h.

For behavioral studies, surrogates and the newly born pups were placed on a reversed 12-hour dark/light cycle (11.00 p.m. light/11.00 a.m. dark). Pups were weaned and group housed on PD21, with each cage containing 2 males and 2 females from the same litter. Only 1 male from each cage was tested for SI. For molecular studies, surrogates and their pups remained on a regular 12-hour cycle (7.00 a.m. light/7.00 p.m. dark). Only male offspring were used for these studies. To avoid the problem of oversampling [21], no more than 1 animal per litter was used for any of the molecular experiments reported. For behavioral experiments a maximum of 2 male pups from each litter were used for all experiments. All experimental protocols were approved by the Weill Cornell Medical College Institutional Animal Care and Use Committee and were in accordance with NIH directives for animal studies.

\section{Social Interaction}

Individual test mice were tested for SI during their dark cycle at three ages (PD25, 35 and 45), time points that correspond to previous studies of SI in adolescent mice [22]; $24 \mathrm{~h}$ prior to the test, 1 male animal from each cage was individually housed (test animal), leaving 1 male (stimulus animal) and 2 females per group housed in their original home cage. After 5 min of habituation to the testing environment, the stimulus mouse was introduced into the cage containing the test animal and the interaction was video recorded for a total of $5 \mathrm{~min}$. Trained raters manually scored the social behavior of the test mouse directed toward the stimulus mouse during the entire 5-min test period. The SI score was derived by adding the duration that the test mouse engaged in the following social activities: investigation of head/neck, flank and anogenital regions of the stimulus mouse, allogrooming and proximity [22]. Between each SI test, both the test and stimulus mice were returned to their original home cage and group housed with the females.

USVs were recorded during all SI tests as previously described [22]. Briefly, an ultrasonic microphone (UltraSoundGate Model CM16; Avisoft Bioacoustics, Berlin, Germany) was suspended just above the inside of the test cage. Recordings were processed through the recording interface UltraSoundGate 116 (Avisoft Bioacoustics) and assessed with vocalization analysis software (SASLab Pro, version 5.2.06; Avisoft Bioacoustics). To analyze recordings, auditory signals below $30 \mathrm{kHz}$ were excluded using a high- 
pass filter followed by a whistle-tracking function to label vocalizations longer than $3 \mathrm{~ms}$ in duration. A trained observer blinded to the experimental conditions visually verified all identified vocalizations.

\section{Chromatin Immunoprecipitation Assay}

Bilateral dissections of the mPFC were performed on wet ice and the tissue was cross-linked in $1 \%$ formaldehyde. Tissue was lysed with a hand-held homogenizer in cell lysis buffer $(100 \mathrm{mM}$ EDTA, $50 \mathrm{~mm}$ Tris- $\mathrm{HCl}, 1 \%$ SDS) containing protease inhibitors and centrifuged at 5,000 rpm. The nuclear pellet was resuspended in a 2:1 ratio of chromatin immunoprecipitation (ChIP) dilution buffer (0.01\% SDS, $1.2 \mathrm{~mm}$ EDTA, $16.7 \mathrm{~mm}$ Tris-HCl, $167 \mathrm{~mm}$ $\mathrm{NaCl}, 1.1 \%$ Triton X-100) and nuclear lysis buffer $(10 \mathrm{mM} \mathrm{NaCl}$, $10 \mathrm{~mm}$ Tris- $\mathrm{HCl}, 3 \mathrm{mM} \mathrm{MgCl}_{2}, 1 \% \mathrm{NP}-40$ ) and fragmented by sonication. Samples were centrifuged at maximum speed and the supernatant was collected and stored at $-80^{\circ} \mathrm{C}$. An aliquot of the fragmented chromatin was set aside as total input control.

ChIP assays were performed using the ChIP-IT Express kit (Active Motif, Carlsbad, Calif., USA) following the protocol supplied by the company. For the ChIP reaction, $100 \mathrm{ul}$ of the fragmented chromatin was mixed with protein $\mathrm{G}$ magnetic beads and $5 \mathrm{ul}$ of antiMeCP2 antibody (Abcam, Cambridge, Mass., USA) or antiacH3K9, 14 antibody (Millipore, Billerica, Mass., USA). The reaction mixture was incubated at $4^{\circ} \mathrm{C}$ for $24 \mathrm{~h}$ on a rotator. The beads were washed with wash buffer and the DNA was immunoprecipitated.

\section{Tissue Collection for $m R N A$ and Protein Analyses}

To measure the constitutive levels of BDNF and egr 1 mRNA and protein, the mice were euthanized by rapid decapitation, whole brains were removed from the skull and bilateral dissections of the $\mathrm{mPFC}$ were performed on dry ice.

\section{Real-Time RT-PCR}

To study the expression of BDNF and egr1 mRNA, total RNA was extracted and CDNA was synthesized as previously published [20]. The relative amount of each transcript of interest present in the sample was measured by quantitative real-time PCR on 24$26 \mathrm{ng}$ of the resulting cDNA using SYBR Green detection (Applied Biosystems, Foster City, Calif., USA), with parameters as previously published [20]. All samples were measured in duplicate and mRNA levels were normalized to $\beta$-actin mRNA to adjust for small differences in input RNA. Primers used for BDNF exons I and IV [23], egr1 [24] and $\beta$-actin [25] were as published, respectively.

For qPCR performed on samples that underwent ChIP, the qPCR parameters used were as in Chahrour et al. [26]. Primers used for BDNF exon IV were previously reported by Chen et al. [17] and for BDNF exon I by Tsankova et al. [27]. Primers for egr1 were designed using MethPrimer (http://www.urogene.org/cgi$\mathrm{bin} / \mathrm{methprimer/methprimer.cgi).} \mathrm{The} \mathrm{primer} \mathrm{sequences} \mathrm{for} \mathrm{egr} 1$ were as follows: forward $5^{\prime}$ CCAGTTGGGAACCAAGGAG $3^{\prime}$ and reverse $5^{\prime}$ GCCCAAATAAGGTCTGTTCC $3^{\prime}$. All samples were run in triplicate and the levels of immunoprecipitated DNA were normalized to the total input control.

\section{BDNF ELISA}

Mature BDNF protein levels were measured using the BDNF Emax ImmunoAssay (ELISA) system (Promega, Madison, Wisc., USA) as previously described [20]. Standard and samples were performed in duplicate.
Western Blot Analyses

Tissue prepared for BDNF ELISA assays was additionally used for EGR1 Western blot analyses; 40 ug of protein was separated on an $8 \%$ gel along with a Kaleidoscope prestained standard (Bio-Rad, Hercules, Calif., USA). Blots were incubated in primary antibody (EGR1 1:400; Cell Signaling, Danvers, Mass., USA; actin 1:20,000, Millipore, Billerica, Mass., USA) for $12-16 \mathrm{~h}$ at $4^{\circ} \mathrm{C}$. Secondary antibody incubations were performed at room temperature in blocking buffer for $1 \mathrm{~h}$ (horseradish peroxidase-linked IgG-conjugated goat anti-rabbit 1:2,500 for EGR1, or horse anti-mouse 1: 30,000 for actin; Vector Laboratories, Burlingame, Calif., USA). Membranes were visualized with Western Lightning Chemiluminescence solution (Perkin Elmer Life Science, Boston, Mass., USA). Optical density from films was analyzed using NIH Image (NIH, Bethesda, Md., USA).

\section{Statistical Analyses}

When analyzing the SI and USV data, a two-way ANOVA (prenatal treatment $\mathrm{x}$ age) was performed and, when significant ( $\mathrm{p}<$ 0.05 ), a Student's t test was performed at each developmental age. The relationships between SI scores and USV counts were determined by fitting a linear regression individually for each treatment group at all developmental time points and evaluated using Pearson's correlation coefficient. Similarly, the analysis of the molecular data was performed using Student's t test, comparing the PSAL and PCOC groups. Before any analyses were done on the behavioral or molecular data, an outlier test was performed and outliers (2 standard deviations from the mean) were removed.

\section{Results}

\section{PCE Increased SI in Offspring at PD25 and 35 but not at PD45}

To understand the impact of PCE on SI during development, PCOC and PSAL animals were subjected to the SI test at three developmental ages. For SI, we found a main effect of prenatal treatment $\left(\mathrm{F}_{1,128}=4.216, \mathrm{p}<0.05\right)$ and an interaction between prenatal treatment and age $\left(\mathrm{F}_{2,128}=3.781, \mathrm{p}<0.05\right)$. Post hoc analyses revealed a significant increase in total time that the PCOC test mouse spent interacting with the stimulus mouse when tested at PD25 and 35 ( $p<0.05$; fig. 1$)$ compared to the PSAL mice. This increase in interaction was calculated as a combination of all the social measures and not attributable to any one specific measure. No such differences in time spent interacting with the stimulus mouse were observed in PCOC mice at PD45 (fig. 1).

\section{PCE Increased Vocalization Rates during SI at PD25, but not at PD35 or 45}

Using a $2 \times 3$ ANOVA, there was a significant effect of test age $\left(\mathrm{F}_{2,126}=11.62, \mathrm{p}<0.0001\right)$, a marginal effect of treatment $\left(\mathrm{F}_{1,127}=3.25, \mathrm{p}=0.074\right)$ and no interaction 


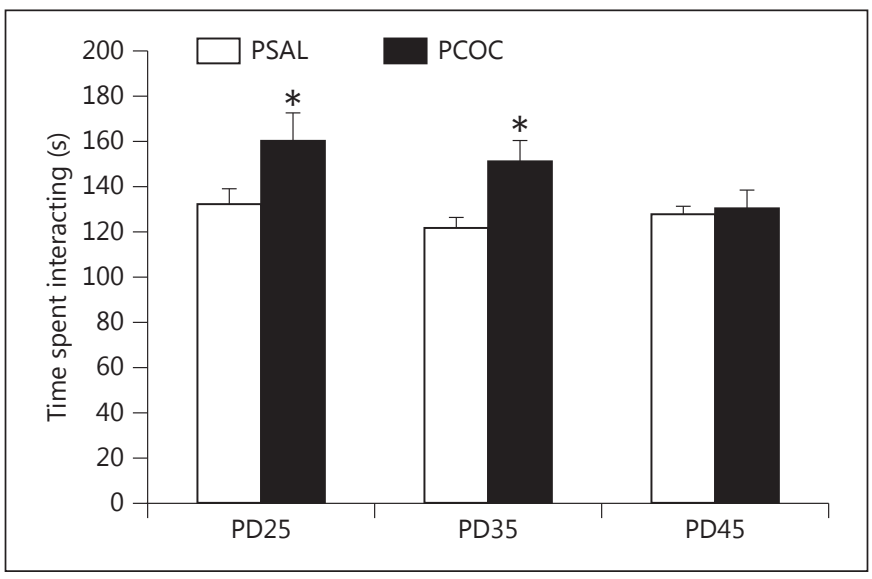

Fig. 1. PCE increased SI in offspring at PD25 and 35 but not at PD45. The total time that the test mouse interacted with the stimulus mouse at each of the developmental ages was measured. Total interaction time was the sum of the time spent on investigation of head/neck, flank and anogenital regions, allogrooming and proximity. There was a significant increase in the total interaction time in the PCOC mice at PD25 and 35 compared to PSAL mice $(* \mathrm{p}<$ 0.05 PCOC vs. PSAL; PD25 PSAL $n=26$, PCOC $n=21$; PD35 PSAL $n=24$, PCOC $n=18$; PD45 PSAL $n=24$, PCOC $n=21$ ). Error bars represent the mean \pm SEM.

between treatment and age $\left(\mathrm{F}_{2,126}=2.36, \mathrm{p}=0.098\right)$. However, using a $2 \times 2$ ANOVA and analyzing the USV data at PD25 and 35 only, we found a main effect of prenatal treatment $\left(\mathrm{F}_{1,85}=14.76, \mathrm{p}=0.0002\right)$, with no effect of age or interaction between prenatal treatment and age. Post hoc analyses revealed a significantly elevated production of USVs in PCOC compared to PSAL mice at PD25 ( $\mathrm{p}<0.01$; fig. 2a), but not when tested at PD35.

When SI scores and USV counts were compared for individual mice, we found that these dependent variables were highly correlated at PD25 for both PCOC $(r=0.73$, $\mathrm{n}=21, \mathrm{p}<0.0005$; fig. $2 \mathrm{c})$ and PSAL mice $(\mathrm{r}=0.7, \mathrm{n}=$ $26, p<0.0001$; fig. 2b). However, these SI scores and USV counts were weakly correlated in PCOC mice at PD35 $(\mathrm{r}=0.48, \mathrm{n}=18, \mathrm{p}<0.05$; data not shown), not correlated in PSAL mice at PD35 $(r=0.23, \mathrm{n}=24, \mathrm{p}=\mathrm{NS})$ and not correlated in either treatment group at PD45 $(\mathrm{r}<$ $0.31, \mathrm{p}=\mathrm{NS})$.

\section{PCE Selectively Increased BDNF Exon IV and egr1}

$m R N A$ Expression in the mPFC of Offspring at PD30

To understand the molecular changes that might contribute to the increase in SI observed in PCOC mice at PD25 and 35, but not at PD45, mRNA levels of BDNF and egr1 were measured in the $\mathrm{mPFC}$ at PD30 and 45. At PD30, there was a significant increase in BDNF exon IV
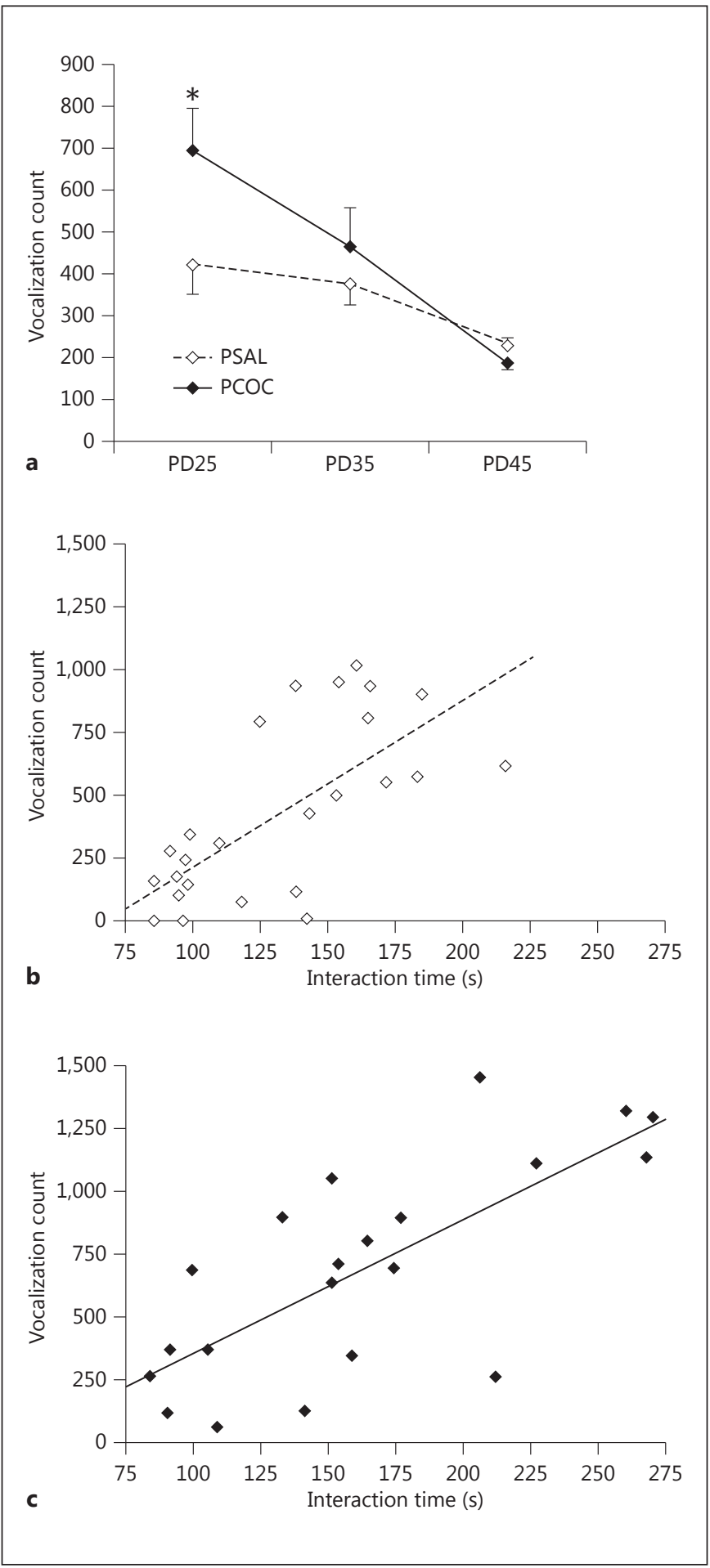

Fig. 2. PCE increased vocalization rates during SI in offspring at PD25, but not at PD35 or 45. The total number of vocalizations produced by PCOC and PSAL social pairs were measured during SI at PD25, 35 and 45. a PCOC mice emitted a greater number of USV s during SI relative to PSAL mice at PD25, but not at PD35 or 45. USV counts were strongly correlated with interaction time at PD25 in both PSAL mice (b) and PCOC mice (c). 


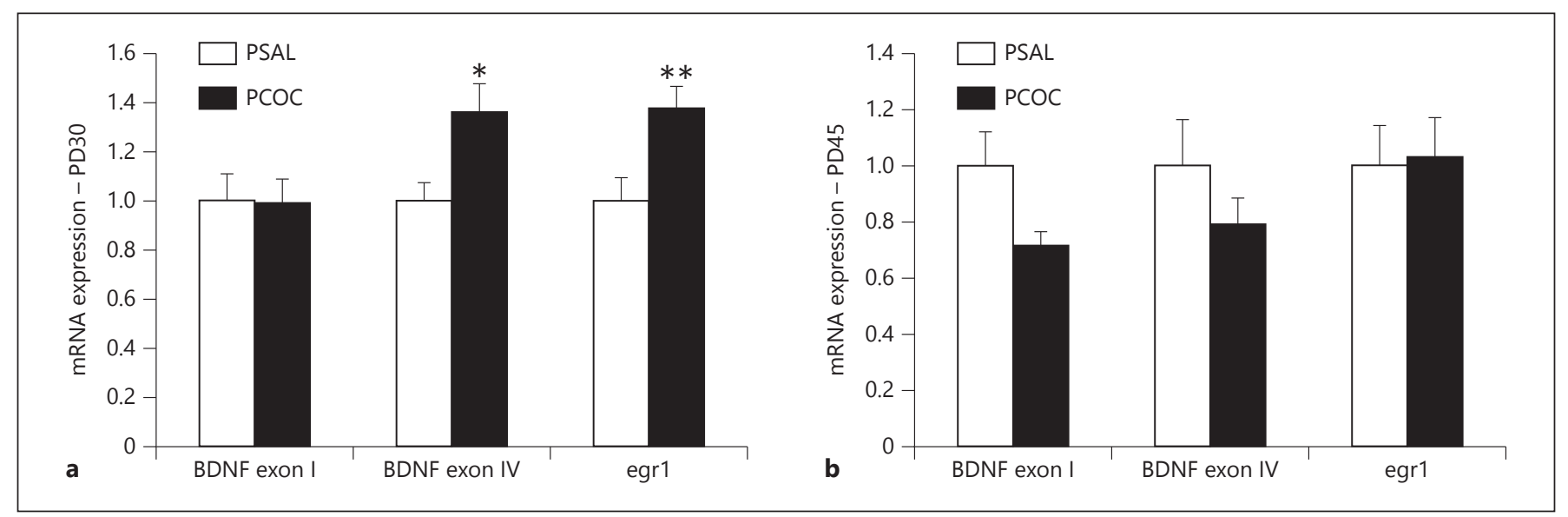

Fig. 3. PCE selectively increased BDNF exon IV and egr1 mRNA expression in the mPFC of offspring at PD30 but not at PD45. Constitutive mRNA levels of BDNF exons I and IV and egr1 were measured in the MPFC of PSAL and PCOC mice at PD30 and 45. a There were increased BDNF exon IV and egr1 mRNA levels in the $\mathrm{mPFC}$ of PCOC mice at PD30 compared to PSAL mice. $\mathbf{b}$ There was no change in the mRNA levels of BDNF exons I and IV or of egr 1 in the mPFC of PCOC mice at PD45 compared to PSAL mice $\left({ }^{*} \mathrm{p}<0.05,{ }^{* *} \mathrm{p}<0.01\right.$, PCOC vs. PSAL; PD30 PSAL $\mathrm{n}=14$, PCOC $\mathrm{n}=13$; PD45 PSAL $\mathrm{n}=6$, PCOC $\mathrm{n}=6$ ). Error bars represent the mean \pm SEM.
Fig. 4. PCE increased EGR1 protein but not BDNF protein in the $\mathrm{mPFC}$ of offspring at PD30. Constitutive protein levels of mature BDNF and EGR1 were measured in the $\mathrm{mPFC}$ of PSAL and PCOC mice at PD30. a There was no change in mature $\mathrm{BDNF}$ protein levels in the $\mathrm{MPFC}$ of PCOC mice compared to PSAL mice. $\mathbf{b}$ There was significantly increased EGR1 protein in the mPFC of PCOC mice compared to PSAL mice $\left({ }^{*} \mathrm{p}<0.05\right.$, PCOC vs. PSAL; PSAL $\mathrm{n}=$ 14$, PCOC $n=13)$. Error bars represent the mean \pm SEM.

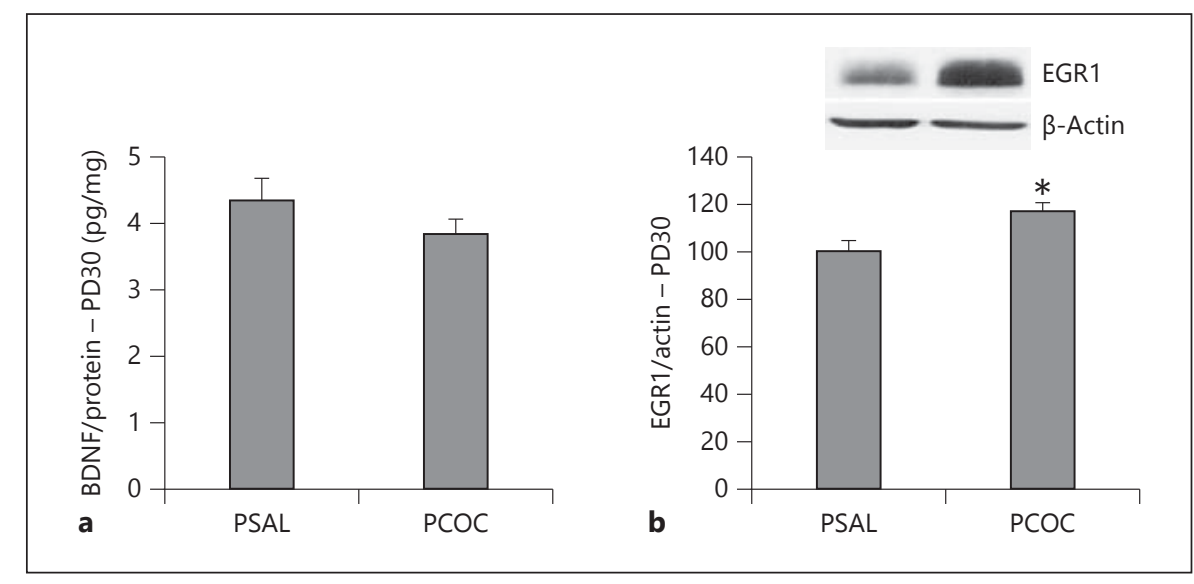

$(\mathrm{p}<0.05)$ and egr $1(\mathrm{p}<0.01)$ mRNA levels with no change in BDNF exon I mRNA levels in the mPFC of PCOC mice (fig. 3a). At PD45 there were no significant differences between prenatal treatment groups for mRNA levels of BDNF exons I and IV and egr1 (fig. 3b).

\section{PCE Increased EGR1 but not BDNF Protein in the $m P F C$ of Offspring at PD30}

To determine whether the increased mRNA levels of BDNF exon IV and egr 1 in the $\mathrm{mPFC}$ of PCOC mice corresponded to an alteration in their protein levels, we measured mature BDNF and EGR1 protein in the mPFC. Because we observed changes in mRNA specifically at PD30 but not at PD45, subsequent molecular experiments were performed only at this earlier age. ELISA was used to measure levels of the mature and functionally active isoform of the BDNF protein and Western blots were used to measure levels of the EGR1 protein. In the mPFC of PCOC mice at PD30, we observed no difference in BDNF protein levels (fig. 4a). However, we did observe a significant increase in EGR1 protein expression $(\mathrm{p}<0.05$; fig. 4b) in the $\mathrm{mPFC}$ of PCOC mice at PD30. These results suggest that PCE-induced enhancement in EGR1 protein levels, but not BDNF protein in the $\mathrm{MPFC}$, may contribute to the increase in SI observed in these animals at PD25 and 35 . 


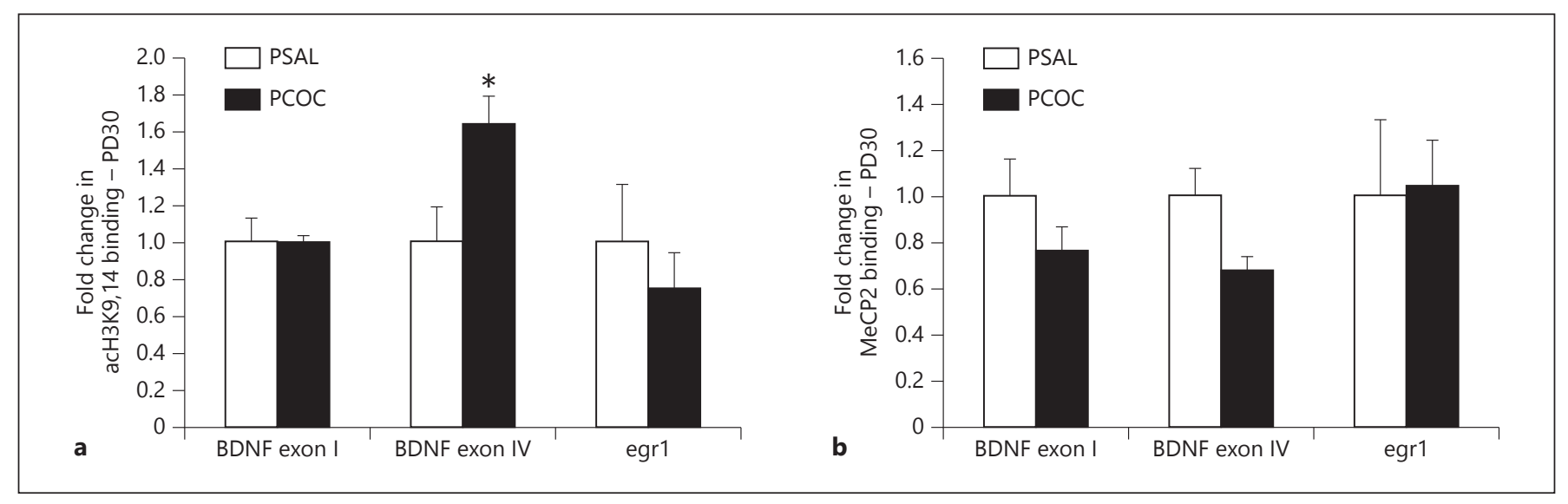

Fig. 5. PCE selectively increased acH3K9,14 and trended towards decreased MeCP2 binding at the BDNF exon IV promoter in the mPFC of offspring at PD30. The association of acH3K9,14 and the binding of MeCP2 at the promoters of BDNF exons I and IV and egrl were identified in the $\mathrm{MPFC}$ of PD30 mice. a There was a significant increase in the association of acH3K9,14 at the promoter

\section{PCE Selectively Increased acH3K9,14 at the BDNF}

Exon IV Promoter in the mPFC of Offspring at PD30

To understand the prenatal cocaine-induced transcriptional regulation of the BDNF and egrl genes that showed increased mRNA levels in the $\mathrm{mPFC}$ at PD30, the association of acH3K9,14 and $\mathrm{MeCP} 2$ to the promoters of these genes was studied. In the $\mathrm{mPFC}$ of PCOC mice at $\mathrm{PD} 30$, there was increased acH3K9,14 ( $\mathrm{p}<0.05$; fig. 5a) and a trend towards decreased $\mathrm{MeCP} 2$ binding $(\mathrm{p}=0.06$; fig. 5b) specifically associated with the promoter of BDNF exon IV, which was consistent with the increased BDNF exon IV mRNA that we observed in the MPFC of PD30 PCOC mice. No changes in acH3K9,14 or MeCP2 binding were observed at the promoter of BDNF exon I (fig. 5a, b).

To identify changes in acH3K9,14 and the binding of $\mathrm{MeCP} 2$ at the promoter of egr1, we first screened the mouse egr 1 promoter region for $\mathrm{CpG}$ islands. Using MethPrimer software (http://www.urogene.org/ methprimer/) $[19,28]$, we identified two CpG islands in the promoter of the egr 1 gene immediately upstream to the transcription start site similar to that identified in the rat [19]. Based on our findings of increased egr1 expression in the mPFC of PCOC mice at PD30, for our ChIP analyses we used primers designed to the more proximal $\mathrm{CpG}$ island in the promoter region of the mouse egr 1 gene, where MeCP2 binding has been thought to act as a transcriptional activator of egr1 expression [19]. We found no change in acH3 $\mathrm{K} 9,14$ or $\mathrm{MeCP} 2$ binding within of BDNF exon IV with no change in acH3K9,14 at the promoters of BDNF exon I and egr1. $\mathbf{b}$ There was a trend $(p=0.06)$ towards a decrease in the binding of $\mathrm{MeCP} 2$ at the promoter of BDNF exon IV with no change in its binding at BDNF exon I and egr $1\left(^{*} \mathrm{p}<\right.$ 0.05 , PCOC vs. PSAL; PSAL $n=8$, PCOC $n=8$ ). Error bars represent the mean \pm SEM.

this $\mathrm{CpG}$ island in the promoter of egr1 in the mPFC of PCOC mice at PD30 (fig. 5a, b).

The findings indicate that PCE can lead to chromatin remodeling marks that persist throughout development in spite of a lack of acute exposure to the drug that can influence gene transcription in a promoter-specific fashion. However, the alterations we investigated do not appear to be the mechanism contributing to the increased egr 1 mRNA expression we observed in the mPFC of PCOC mice at PD30.

\section{Discussion}

In this study we identified increased SI in PCOC mice at PD25 and 35 that normalized by PD45, together with increased USVs specifically at PD25. At the molecular level, we observed increased egr $1 \mathrm{mRNA}$ and protein in the $\mathrm{mPFC}$ of PD30 PCOC mice that also normalized by $\mathrm{PD} 45$, suggesting that the elevated constitutive egr 1 expression in the MPFC of PCOC mice may be mediating the increased SI in these animals. As such, we provide evidence of a novel developmental alteration in SI behavior of PCOC mice coordinate with a possible molecular mechanism underlying the behavior. Furthermore, this is the first study to identify epigenetic modifications in the $\mathrm{mPFC}$ of PCOC mice as early as PD30. However, as we did not identify specific changes in the binding of $\mathrm{MeCP} 2$ or the association of acH3K9,14 at the egr 1 promoter in 
the mPFC of PCOC mice, our findings suggest that other epigenetic marks on the egr1 promoter may be altered following prenatal exposure that impact the developmental expression of this gene, or that other mechanisms are operative.

The social behaviors of PCOC and PSAL mice differed markedly during early adolescence (PD25) but not later (PD45). While we found that PCE enhances SI in mice, other studies that assessed rat SI showed that these behaviors are either insensitive to PCE [9] or are reduced by exposure [6]. There are several possible explanations for the differences between our results and findings of these earlier reports, including differences in species tested, dose or timing of exposure, and behavioral test methodology. In the present study, test mice were socially isolated for $24 \mathrm{~h}$ prior to the test, whereas in studies using rats [6, 9] test animals were not isolated prior to behavioral testing. The duration of social isolation prior to testing influences SI response in mice [29] and rats [30] and may be important for capturing the effects of PCE on social behavior. Furthermore, rat subjects were tested under low white light in a novel environment $[6,31]$, whereas our behavioral tests were conducted in the home cage during the dark cycle under red light conditions, which provides optimal conditions for SI among adolescent mice [22, 29, 32].

Although the SI test is widely used for assessing social deficits, abnormalities in the rodent responses are difficult to interpret. For instance, the fragile - X knockout mouse, one of the most extensively studied genetic models of a disability that features social deficits, can express greater levels of SI than its wild-type controls [33, 34]. While the direction of this difference is unexpected, it might be attributed to a loss in social regulation [35]. Likewise, the increased SI scores of early adolescent mice exposed in utero to cocaine might also indicate impaired social regulation. These results might also suggest less inhibition and an increased likelihood of risk-taking behavior in adolescent mice following PCE. Furthermore, PCE increases frontal cortex reactivity to stress [36] and environmental changes [37] that are likely to accompany social isolation. From this perspective, one might consider the possibility that PCOC mice may be especially sensitive to the enhancing effect of short-term isolation on social approach, rendering them more likely to engage in longer bouts of SI. In this regard, elucidating the interactions between PCE, egrl expression and frontal cortex reactivity might help explain why behavioral differences between experimental and control groups are lost later in maturation; declining levels of egr 1 in late adolescent
PCOC mice might normalize sensitivity to the short period of isolation and restore SI to normal levels. Other behavioral tests, such as social conditioned place preference $[22,35]$ and empathy testing $[38,39]$, might also elucidate the underlying psychological experiences that contribute to abnormal SI.

Our results at PD25 are consistent with other studies that have shown increased vocalization rates associated with more robust SI responses [22, 40]. This association has been extensively characterized for B6 mice during early adolescence [22]. Interestingly, the vocalization rate of PD25 PCOC mice is twice that of age-matched saline controls $(\mathrm{PCOC}=701.15$, PSAL $=427.77)$. However, despite the large difference in vocalizations between treatment groups at $\mathrm{PD} 25$, this difference disappears with age: SI of PCOC animals at PD35 remains elevated above saline controls. Perhaps SI is more sensitive to prenatal injury from cocaine exposure than is vocalization rate. Alternatively, recovery of vocalization rates in PCOC adolescent maturation may simply precede recovery of SI behavior.

Social behavior is sensitive to changes in egr 1 and ERK2 expression within the mPFC $[12,13]$ gene products that are downstream of BDNF [15]. We identified increased levels of EGR1 protein but not BDNF protein in PCOC $\mathrm{mPFC}$ at PD30 compared to PSAL mice, suggesting that these EGR1 effects may be BDNF independent. Since egr1 is an immediate early gene, its expression is downstream of several other signaling pathways. Further experiments will need to be conducted to identify the specific upstream signaling pathways mediating the increased egr 1 levels observed in the MPFC of PCOC mice. Furthermore, our results suggest that the expression of egr 1 and not BDNF in the mPFC of PCOC mice may contribute to the change in SI observed in these animals at this age. To establish a direct causal role of egr 1 would require demonstration of a normalizing of behavior following infusion of shRNA directly into the mPFC of PCOC mice before the SI test, a technique that has been performed in adult rats [13].

We identified increased mRNA levels of the activitydriven transcripts including BDNF exon IV and egr1 in the $\mathrm{mPFC}$ of PCOC mice at PD30. However, we did not observe any difference in the mRNA expression of BDNF exon I, suggesting a transcript-specific effect of PCE in the mPFC. Of particular interest was the normalization of the expression of these transcripts between PD30 and 45 in the $\mathrm{mPFC}$ of PCOC mice. This is in agreement with our recent findings of unaltered constitutive levels of BDNF exons I and IV in the MPFC of adult PCOC mice [41]. 
Both BDNF and egr1, which are dynamic markers of synaptic activity, have increased expression during critical periods of early brain development, when these genes play a crucial role in synaptic maturation including the pruning of unnecessary connections. However, later in adolescence and adulthood, the expression of these genes decreases [42-44]. Thus, the increased expression of these transcripts in the MPFC of PD30 PCOC mice may suggest a delay in the maturation of this region. A similar phenomenon was observed in the ventral tegmental area (VTA) of PCOC animals in which there was a significant delay in the switch from the immature calcium-permeable AMPA and NMDA receptor subunits to the mature calcium-impermeable receptor subunits [45]. One possible explanation of our findings is that the delayed glutamatergic maturation in the VTA [45] could feed-forward via its strong dopaminergic projections to the $\mathrm{mPFC}$ and impair the development of this region via dysmaturation of dopaminergic signaling.

We were unable to establish whether the epigenetic modifications we identified in the promoter regions of $\mathrm{BDNF}$ exon IV regulate the transcription of these genes. In the mPFC of PD30 PCOC mice we found increased acH3 K9,14 and a trend towards decreased MeCP2 binding, specifically at the promoter of BDNF exon IV. Such findings are analogous to epigenetic changes seen as increased rates of DNA methylation of BDNF exon VI, which are evident in adolescents born to human cigarette smokers [46]. However, we did not observe any change in $\mathrm{MeCP} 2$ binding or the association of acH3K9,14 at the promoter of egr 1 , in spite of observing an increase in its expression. These results suggest that PCE alters the epigenetic marks at specific promoter regions within the
mPFC that are evident as early as PD30 and may impact other epigenetic marks, including DNA methylation, that mediate the dysregulated egr1 expression observed in these animals.

To conclude, this study suggests that PCE results in a transient increase in SI during early adolescence, potentially due to a coincident upregulation of egr 1 expression in the $\mathrm{mPFC}$ that engenders greater sensitivity to isolation distress. These results may suggest that targeted molecular and behavioral treatments during critical periods of brain development could normalize function in a region that plays a crucial role in cognitive maturation. Furthermore, the gradual disappearance of differences in social behavior between cocaine-exposed and control groups warrants consideration. Critically, early social experience can shape brain development and influence adult social functioning $[28,47]$. Thus, the possibility of impaired adolescent social functioning following PCE may be highly relevant to other adult social behaviors and might suggest possible use of social interventions to normalize child development and subsequent social maturation following such exposures.

\section{Acknowledgment}

This research was supported by NIH-NIDA grants DA017905 and DA00354 to Dr. Barry Kosofsky and NIH-NIDA grant DA02254-05 to Dr. Garet P. Lahvis.

\section{Disclosure Statement}

The authors report no financial interests or conflicts of interest.

\section{References}

1 Sobrian SK, Holson RR: Social behavior of offspring following prenatal cocaine exposure in rodents: a comparison with prenatal alcohol. Front Psychiatry 2011;2:66.

2 Blakemore SJ: The developing social brain: implications for education. Neuron 2010;65: 744-747.

3 Oro AS, Dixon SD: Perinatal cocaine and methamphetamine exposure: maternal and neonatal correlates. J Pediatr 1987;111:571578.

4 Tronick EZ, et al: Cocaine exposure is associated with subtle compromises of infants' and mothers' social-emotional behavior and dyadic features of their interaction in the faceto-face still-face paradigm. Dev Psychol 2005; 41:711-722.
5 Delaney-Black V, et al: Teacher-assessed behavior of children prenatally exposed to cocaine. Pediatrics 2000;106:782-791.

6 Overstreet DH, et al: Enduring effects of prenatal cocaine administration on emotional behavior in rats. Physiol Behav 2000;70:149-156.

7 Estelles J, et al: Prenatal cocaine exposure alters spontaneous and cocaine-induced motor and social behaviors. Neurotoxicol Teratol 2005;27:449-457.

8 Johns JM, Noonan LR: Prenatal cocaine exposure affects social behavior in Sprague-Dawley rats. Neurotoxicol Teratol 1995;17:569-576.

-9 Magalhaes A, et al: Prenatal exposure to cocaine and enriched environment: effects on social interactions. Ann NY Acad Sci 2006; 1074:620-631.
10 Gonzalez LE, et al: Medial prefrontal transection enhances social interaction. I. Behavioral studies. Brain Res 2000;887:7-15.

11 van Kerkhof LW, et al: Social play behavior in adolescent rats is mediated by functional activity in medial prefrontal cortex and striatum. Neuropsychopharmacology 2013;38: 1899-1909.

12 Carrier N, Kabbaj M: Sex differences in social interaction behaviors in rats are mediated by extracellular signal-regulated kinase 2 expression in the medial prefrontal cortex. Neuroscience 2012;212:86-92.

13 Stack A, et al: Sex differences in social interaction in rats: role of the immediate-early gene zif268. Neuropsychopharmacology 2010;35: $570-580$.
Social Development in Prenatal

Cocaine-Exposed Mice
Dev Neurosci 2014;36:338-346 DOI: $10.1159 / 000360524$ 
14 Schmidt HD, Duman RS: Peripheral BDNF produces antidepressant-like effects in cellular and behavioral models. Neuropsychopharmacology 2010;35:2378-2391.

$\checkmark 15$ Rosenblum K, et al: The role of extracellular regulated kinases I/II in late-phase long-term potentiation. J Neurosci 2002;22:5432-5441.

16 Martinowich K, et al: Activity-dependent brain-derived neurotrophic factor expression regulates cortistatin-interneurons and sleep behavior. Mol Brain 2011;4:11.

17 Chen WG, et al: Derepression of BDNF transcription involves calcium-dependent phosphorylation of MeCP2. Science 2003;302: 885-889.

18 Tian F, Marini AM, Lipsky RH: NMDA receptor activation induces differential epigenetic modification of BDNF promoters in hippocampal neurons. Amino Acids 2010;38: 1067-1074.

19 Gupta S, et al: Histone methylation regulates memory formation. J Neurosci 2010;30: 3589-3599.

20 Kabir ZD, et al: Brain-derived neurotrophic factor genotype impacts the prenatal cocaineinduced mouse phenotype. Dev Neurosci 2012;34:184-197.

-21 Holson RR, Pearce B: Principles and pitfalls in the analysis of prenatal treatment effects in multiparous species. Neurotoxicol Teratol 1992;14:221-228.

22 Panksepp JB, et al: Affiliative behavior, ultrasonic communication and social reward are influenced by genetic variation in adolescent mice. PLoS One 2007;2:e351

23 Hong EJ, McCord AE, Greenberg ME: A biological function for the neuronal activity-dependent component of BDNF transcription in the development of cortical inhibition. Neuron 2008;60:610-624.

24 Tyan SW, et al: Serum- and glucocorticoidinducible kinase 1 enhances zif268 expression through the mediation of SRF and CREB1 associated with spatial memory formation. J Neurochem 2008;105:820-832.
25 Giordano TP 3rd, et al: Up-regulation of dopamine $\mathrm{D}_{2} \mathrm{~L}$ mRNA levels in the ventral tegmental area and dorsal striatum of amphetamine-sensitized C57BL/6 mice: role of $\mathrm{Ca}_{\mathrm{v}} 1.3$ L-type $\mathrm{Ca}^{2+}$ channels. J Neurochem 2006;99:1197-1206.

26 Chahrour M, et al: MeCP2, a key contributor to neurological disease, activates and represses transcription. Science 2008;320:1224-1229.

27 Tsankova NM, Kumar A, Nestler EJ: Histone modifications at gene promoter regions in rat hippocampus after acute and chronic electroconvulsive seizures. J Neurosci 2004;24:56035610 .

28 Hammock EA, Levitt P: The discipline of neurobehavioral development: the emerging interface of processes that build circuits and skills. Hum Dev 2006;49:15.

29 Panksepp JB, et al: Differential entrainment of a social rhythm in adolescent mice. Behav Brain Res 2008;195:239-245.

30 Niesink RJ, van Ree JM: Short-term isolation increases social interactions of male rats: a parametric analysis. Physiol Behav 1982;29: 819-825.

31 File SE: The use of social interaction as a method for detecting anxiolytic activity of chlordiazepoxide-like drugs. J Neurosci Methods 1980;2:219-238.

32 McFarlane HG, et al: Autism-like behavioral phenotypes in BTBR $\mathrm{T}+\mathrm{tf} / \mathrm{J}$ mice. Genes Brain Behav 2008;7:152-163.

33 Spencer CM, et al: Altered anxiety-related and social behaviors in the Fmr1 knockout mouse model of fragile X syndrome. Genes Brain Behav 2005;4:420-430.

34 Spencer CM, et al: Social behavior in Fmr1 knockout mice carrying a human FMR1 transgene. Behav Neurosci 2008;122:710715.

35 Lahvis GP, Alleva E, Scattoni ML: Translating mouse vocalizations: prosody and frequency modulation. Genes Brain Behav 2011;10:416.

36 Elsworth JD, Morrow BA, Roth RH: Prenatal cocaine exposure increases mesoprefrontal dopamine neuron responsivity to mild stress. Synapse 2001;42:80-83.
37 Morrow BA, Elsworth JD, Roth RH: Male rats exposed to cocaine in utero demonstrate elevated expression of Fos in the prefrontal cortex in response to environment. Neuropsychopharmacology 2002;26:275-285.

38 Chen Q, Panksepp JB, Lahvis GP: Empathy is moderated by genetic background in mice. PLoS One 2009;4:e4387.

-39 Panksepp JB, Lahvis GP: Rodent empathy and affective neuroscience. Neurosci Biobehav Rev 2011;35:1864-1875.

40 Scattoni ML, Ricceri L, Crawley JN: Unusual repertoire of vocalizations in adult BTBR $\mathrm{T}+\mathrm{tf} / \mathrm{J}$ mice during three types of social encounters. Genes Brain Behav 2011;10:4456.

41 Kabir ZD, Katzman AC, Kosofsky BE: Molecular mechanisms mediating a deficit in recall of fear extinction in adult mice exposed to cocaine in utero. PLoS One 2013; 8:e84165.

42 Aid T, et al: Mouse and rat BDNF gene structure and expression revisited. J Neurosci Res 2007;85:525-535.

43 Greenberg ME, et al: New insights in the biology of BDNF synthesis and release: implications in CNS function. J Neurosci 2009;29: 12764-12767.

44 Knapska E, Kaczmarek L: A gene for neuronal plasticity in the mammalian brain: Zif268/ Egr-1/NGFI-A/Krox-24/TIS8/ZENK? Prog Neurobiol 2004;74:183-211.

45 Bellone C, Mameli M, Luscher C: In utero exposure to cocaine delays postnatal synaptic maturation of glutamatergic transmission in the VTA. Nat Neurosci 2011;14:1439-1446.

46 Toledo-Rodriguez M, et al: Maternal smoking during pregnancy is associated with epigenetic modifications of the brain-derived neurotrophic factor-6 exon in adolescent offspring. Am J Med Genet B Neuropsychiatr Genet 2010;153B:1350-1354.

47 Zahn-Waxler C, Radke-Yarrow M: The origins of empathic concern. Motiv Emot 1990; 14(2):107-130. 